\title{
Crystal engineering: A brief overview
}

\author{
GAUTAM R DESIRAJU \\ Solid State and Structural Chemistry Unit, Indian Institute of Science, Bangalore 560012 \\ e-mail: desiraju@sscu.iisc.ernet.in
}

\begin{abstract}
Crystal structures of organic and metal-organic compounds have been determined in enormous numbers over the past century, and at the time of writing this review, the Cambridge Structural Database has just crossed the half million mark. The possibility of designing a particular crystal packing is, however, of more recent origin and the subject of crystal engineering has addressed this possibility, more or less systematically, during the past 30 years. Crystal engineering demands a detailed and thorough knowledge of intermolecular interactions, which act as the supramolecular glue that binds molecules into crystals. It also requires systematic strategies for the design of a crystal, the architectural blueprint as it were. Finally, this enterprise needs to be geared towards a useful property in that the crystal that is being designed is a functional one. All these features of the subject are directly or indirectly connected with the fact that there is a very large database of known crystal structures that is available to the crystal engineer. This review attempts to briefly survey the current scenario in this expanding subject.
\end{abstract}

Keywords. Crystallography; crystal structure; hydrogen bond; polymorphism; pharmaceuticals.

\section{Introduction}

Crystal engineering is concerned with the construction of crystal structures of organic and metal organic species, using design principles that are derived from an understanding of the intermolecular interactions that prevail in molecular solids. ${ }^{1}$ Ideally, the crystal to be designed has a specific function whether it is chemical reactivity of a desired kind, an optical, magnetic or electronic property, or still the ability to absorb within the crystal another small molecule. ${ }^{2-4}$ To summarise, crystal engineering is the rational design of functional molecular solids. This subject has been in active investigation for around 30 years. ${ }^{5}$ The conceptual origins of crystal engineering go much further back in time and indeed right back to the origins of X-ray crystallography when Bragg noted similarities between the crystal structures of naphthalene and anthracene. ${ }^{6}$ In the modern context, the concepts of crystal engineering are applicable to any kind of intermolecular assembly whether it is protein ligand recognition, the design of supramolecular polymers, systems for drug delivery or the study of organized media other than crystalline solids. Crystal engineering is accordingly of very wide scope and it has brought together investigators from many disciplines. ${ }^{7}$ These include organic chemistry, inorganic chemistry, physical chemistry, X-ray crystallography, materials sciences and computational chemistry.

The first phase of this subject is exemplified by the work of Robertson who was perhaps the first to systematically relate molecular structure of organic compounds with their crystal structure. ${ }^{8}$ Robertson surveyed the crystal structures of a large number of polynuclear aromatic hydrocarbons in the 1940s and 1950 s. The determination of a crystal structure was an extremely difficult task in those days and Robertson's crystallographic investigations are of an almost heroic nature. He divided these flat aromatic molecules into two categories: in the first, the molecular thickness is comparable to the length and breadth; in the second the thickness is small in comparison with the two other dimensions. Each of these categories was associated with a certain type of crystal structure - the former with what we would now term the herringbone packing displayed by naphthalene and the latter with the stacked structure as exemplified by coronene. Although this work was carried out 60 years ago, its consequences impinge on the subject of crystal engineering even today because the essential question in crystal engineering remains 'Given the molecular structure of an organic compound, what is its crystal structure?' In other words how do molecules recognize one another during the crystallization event? This question is easy 
to phrase but it is extremely difficult to answer. At the present time there is no general answer to this question.

Indeed this question can be sub-divided into several smaller questions as shown in the following scheme:

$$
\text { One } \rightarrow \text { Few } \rightarrow \text { Many } \rightarrow \text { Crystal. }
$$

The fact is that a crystal is built up from a large number of molecules and this building up (aufbau) need not be a smooth and continuous process in which the final structure of the crystal is established and is recognizable from the very initial stages of molecular assembly. A few molecules assemble to form a cluster; several clusters may assemble to form larger entities and these entities interact with each other through an event that we term as nucleation, which leads in turn to the first crystal. Local order and organization within the small and then the larger clusters must lead eventually to long range periodicity that one associates with the crystalline state. ${ }^{9}$ However, one does not know when and how this happens. A small cluster need not necessarily be a good model for a larger cluster and a large cluster in turn may not necessarily be a good model for the even larger clusters. In other words, there may be discontinuities in the process of building up of clusters that leads to nucleation and growth of a crystal. Therefore, the observation and understanding of the molecule-to-crystal progression is a difficult task and there are no easy answers. X-Ray crystallography provides information on the final crystal structure of a molecule. At the present time, the structural chemist is restricted to the knowledge of the structures of just the starting molecule and the final crystal. One does not know the structures of the species during the intermediate stages of aggregation in any great detail although certain lines of research are beginning to cast some light on these interesting transitory stages of the supramolecular reaction that we call crystallization. ${ }^{10-13}$

Systematic crystal engineering was launched with the contributions of Schmidt in the Weizmann Institute. The contributions from this group in the 1950s and 1960 s, on the solid state photochemical reactivity of trans-cinnamic acids and other alkenes, laid the foundation of the subject. ${ }^{14}$ Schmidt is usually credited with having introduced the term crystal engineering into the chemical literature although there is an earlier report by Pepinsky wherein the term is actually mentioned for the very first time. ${ }^{15}$
The work in the Weizmann Institute was followed, after the death of Schmidt in 1972, by other contributions from Israel most notably from the groups of. Cohen, ${ }^{16}$ Herbstein, ${ }^{17}$ Lahav and Leiserowitz. ${ }^{18}$ These developments were paralleled by work of Curtin and Paul from the University of Illinois on phase transitions, gas-solid reactions, crystal polarity and polymorphism. ${ }^{19}$ Also notable in the early years is work from the group of Wegner and Enkelmann on diacetylene polymerization ${ }^{20}$ and from the University of Cambridge on topotaxy and defect controlled topochemistry ${ }^{21}$ (Thomas and Jones). All these early studies are of outstanding importance and allowed the subject of crystal engineering to gain some sort of identity thereby attracting the interest of crystallographers, chemists and materials scientists to its ranks.

The next phase of crystal engineering may be said to have begun in the late 1980s. Typical contributions of this period include a paper by Ermer on the crystal structure of adamantane-1,3,5,7-tetracarboxylic acid. ${ }^{22}$ The novel analysis of the complex packing of this molecule in the crystal, in terms of interpenetrated networks, is the forerunner of the topological approach to the description of crystal structures of molecular solids. Continuing on this theme, a paper in 1990 by Robson, on interpenetration as a theme in the crystal structures of some coordination compounds, is of fundamental importance and was the starting point of investigations on coordination polymers ${ }^{23}$ which in turn has led to identification of some of these compounds as metal organic framework structures (MOF). ${ }^{24}$ The study of coordination polymers is a vast enterprise today and numerically speaking, there are probably more workers in this branch of crystal engineering than in any other. Restrictions of length prevent one from presenting a more detailed discussion on this vital aspect of the subject.

A paper by Desiraju and Gavezzotti in 1989 entitled 'From molecular to crystal structure; "polynuclear aromatic hydrocarbons' echoes Robertson's work in a more quantitative manner and identified the molecule $\rightarrow$ crystal transformation as the key issue in crystal engineering. ${ }^{25}$ The appearance of a book by Desiraju in 1989 entitled 'Crystal engineering: The design of organic solids' showed that the subject of crystal engineering goes far beyond organic solid state reactivity. ${ }^{1}$ This book is still the only single author book on the subject. Desiraju defined crystal engineering as "the understanding of 
intermolecular interactions in the context of crystal packing and in the utilisation of such understanding in the design and new solids with desired physical and chemical properties'. This definition seems to have stood the test of time and the subject today includes three distinct activities which form a continuous consequence (i) the study of intermolecular interactions; (ii) the study of packing modes in the context of these interactions with the aim of designing a strategy for crystal construction and; (iii) the study of crystal properties. These three stages represent the what, the how and the why of crystal engineering. ${ }^{26}$ The final development in the kick offstage of crystal engineering was the identification of an organic crystal as a supramolecular par excellence by Dunitz in 1991. ${ }^{27}$ Dunitz based his argument on the paradigm of supramolecular chemistry which was recognized just few years earlier, in 1987, through the award of a Nobel Prize to Pedersen, Cram and Lehn.

\section{Supramolecular retrosynthesis}

The central dogma of supramolecular chemistry was enunciated by Lehn who stated that just as a molecule is built with atoms by connecting them with covalent bonds, a supramolecule is built up with molecules using intermolecular interactions as connectors. $^{28}$ The idea of molecular recognition therefore became closely integrated with supramolecular chemistry. Taking up from Dunitz's concept of a crystal as a supramolecular entity, Desiraju suggested that crystal engineering, the design and construction of a molecular crystal, is a form of solid state supramolecular synthesis. ${ }^{29}$ Continuing these analogies further, a polymorph of an organic solid is the supramolecular equivalent of what a structural isomer is in molecular chemistry, ${ }^{30}$ crystallization becomes a supramolecular reaction and the crystal nucleus may be likened to a transition state. ${ }^{12}$ It was in the middle 1990 s that the concept of crystal engineering entered the mainstream of organic chemistry. Three definitive reviews appeared in this and related contexts: Whitesides described non-covalent synthesis as a way of using physical organic chemistry to make molecular aggregates; ${ }^{31}$ Desiraju defined supramolecular synthons as structural units within crystals that can be formed by known or conceivable synthetic operations (scheme 1); ${ }^{29}$ Stoddart defined two types of supramolecular synthesis, ${ }^{32}$ the first is more akin to
Desiraju's definition of crystal engineering and involves a building up of supramolecular entities with intermolecular interactions; the second utilizes supramolecular synthesis as an aid to the building up of exotic molecular entities, like rotaxanes, catenanes and more recently exotic topological shapes like Borromean rings. ${ }^{33}$

Supramolecular synthons are kinetically defined structural units that express the core features of a crystal structure and which encapsulate the essence of crystals in terms of molecular recognition. Synthons consist of molecular fragments and the supramolecular associations between them such as hydrogen bonds and other directional interactions. We have already referred to the crucial problem in crystal engineering, namely that a smaller cluster need not be the best model for a larger cluster indeed an important assumption in synthon theory is that the supramolecular synthon is a reasonable approximation to the entire crystal despite the simplifications that are inherent, and indeed necessary, in its definition.

The main aim of crystal engineering is to make particular crystal structures from particular molecules. Unfortunately, crystal structures do not correspond in a straightforward manner to molecular functionality. This lack of modularity is a serious issue. In the language of complex systems, one states that a crystal structure is an emergent property of molecules. Because the synthetic step in crystal engineering is not always straightforward, one of the practical aims of the subject is to identify similar molecules that lead to similar crystal constructs (identify at least those systems, where an element of modularity is present when going from molecule $\rightarrow$ crystal). If a certain molecular similarity also leads to packing similarity in the form of similar supramolecular synthons, then the use of other similar molecules is also expected to lead to predictable crystal structures. Considering compounds 1 through 4 , one notes that $\mathbf{1}$, hydrogen cyanide, is the prototype for a linear chain based on $\mathrm{C}-\mathrm{H} \cdots \mathrm{N}$ hydrogen bonds (scheme 2). ${ }^{34}$ The crystal structure was determined more than 50 years ago and is from the classical era. ${ }^{35}$ Introduction of extra hydrocarbon groups between the cyano and ethynyl groups in compound 1 furnishes compounds 2 through 4 . Introduction of a $\mathrm{C} \equiv \mathrm{C}$ group between cyano and ethynyl groups gives 2, cyanoacetylene. However, this extra acetylene functionality has little effect on the linear chain of molecules that is formed. Once 


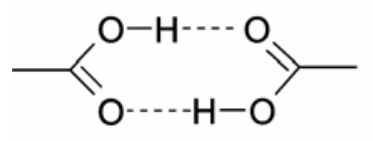

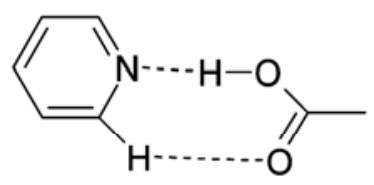<smiles></smiles>

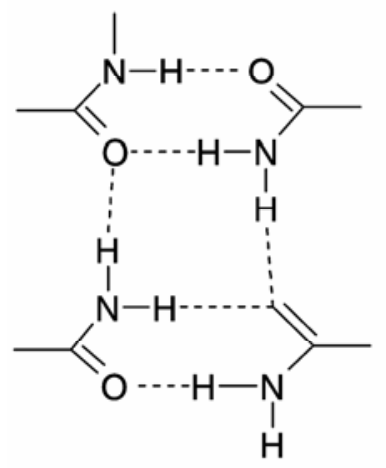<smiles>O=C1CCCC(=O)N1[TlH]I</smiles><smiles>C#CC1ON(C)O1</smiles>

Scheme 1. Representative supramolecular synthons.

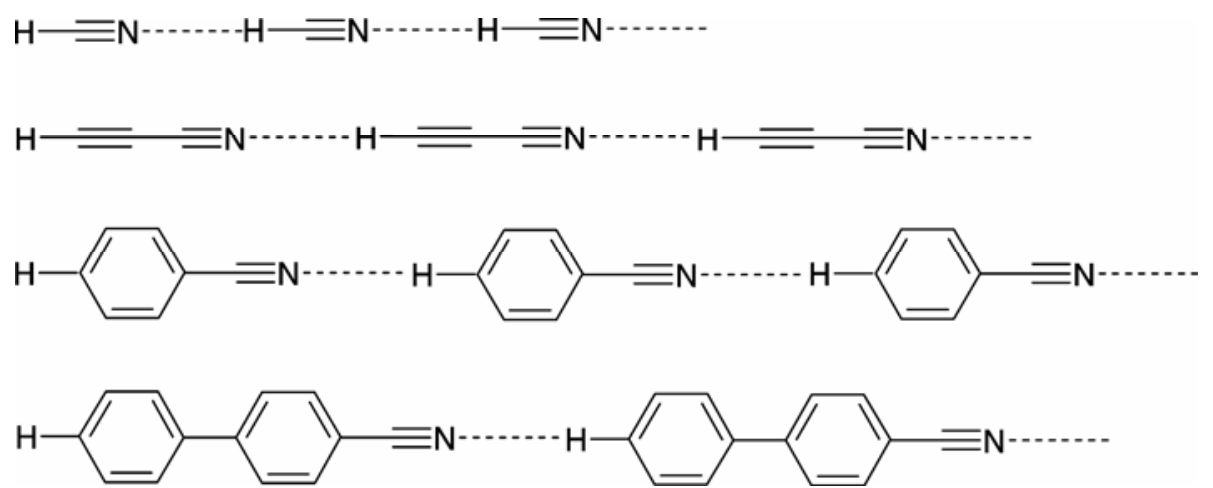

Scheme 2. Supramolecular retrosynthesis.

again the $\mathrm{C}-\mathrm{H} \cdots \mathrm{N} \equiv \mathrm{C}$ hydrogen bond is seen. In $\mathbf{3}$, 4-ethynylbenzonitrile, the group that intervenes between the cyano and ethynyl groups is a 1,4disubstituted phenyl ring. Once again there is no change in the type of linear chain pattern that is formed with $\mathrm{C}-\mathrm{H} \cdots \mathrm{N} \equiv \mathrm{C}$ bonds. Finally, in 4, 4-ethynyl-4'-cyanobiphenyl, the phenyl ring in $\mathbf{3}$ is replaced by a $4,4^{\prime}$-disubstituted biphenyl fragment. We note that the linear chain with $\mathrm{C}-\mathrm{H} \cdots \mathrm{N} \equiv \mathrm{C}$ hydrogen bonds is conserved in all four crystal structures. The nature of the hydrocarbon fragment that comes between the cyano and ethynyl groups has little effect on the formation of the supramolecular linear chain. In other words it may be reasonably expected that other molecules that contain ethynyl and cyano functionalities, preferably at $180^{\circ}$ to each other, will also form the same kind of linear chain. Alternatively, one might say that the hydrocarbon functionalities in these molecules are structurally well insulated from the cyano and ethynyl groups. Such molecules (1 through 4) are well-suited for crystal engineering applications because the crystal structures lend themselves easily to retrosynthetic analysis with supramolecular synthons. This text book example provides a good illustration as to how synthon theory is being applied to real systems in crystal engineering.

\section{The synthon as a simplifier of crystal structures}

Modular behaviour as described above for ethynyl cyanobenzenes is not the norm for organic molecules. Crystal structures are of course related to molecular structures, but this relationship is generally not a simple one. The behaviour of a functional 
group in a molecule during crystallization depends upon the nature and positioning of all the other functional groups in the molecule. Crystal structures are emergent properties. To complicate matters further, hydrocarbon residues, which are generally not regarded as functional groups in the molecular chemistry, certainly count as functionalities in supramolecular chemistry and crystal engineering. Therefore our understanding of the molecule $\rightarrow$ crystal paradigm in crystal engineering is not at all straightforward. A simplification is required and is furnished through the supramolecular synthon. The general idea is that a good or robust synthon is one which appears in a large number of cases wherein a particular set of molecular functionalities is present. If the same set of molecular functionalities is present in another molecule then it is expected that the same supramolecular synthon will appear. Ideally, even if other functional groups are present they will not interfere with the appearance of the supramolecular synthon under discussion. To paraphrase, certain functionalities are more critical to the appearance of certain structural features in the final structures. The synthon is the device through which information content passes from molecular structure to crystal structure. It is a means of simplifying a crystal structure. The synthon is a model for the entire crystal structure and it is one which is hopefully representative of the complete crystal. The entire arena is an energy and structural space of great complexity with local energy minima connected by several intersecting and non-intersecting paths. Good synthons help one to traverse this landscape with maximum efficiency. Other methods of structure simplification have also been proposed. Gavezzotti has used space group information to generate small clusters of molecules. ${ }^{36}$ Etter considered only strong hydrogen bonds to reduce crystal structures down to the topology of these interactions. ${ }^{37}$ The synthon model is in contrast of the two modes of simplification mentioned above. It is a probabilistic model and is concerned with the frequency of occurrence of sub-structural units which are representative not only of the final crystal but also of patterns that evolve during crystallization. If a pattern is noticed often enough it is assumed to be kinetically favoured and is likely to recur in other crystals of related molecules. Crystallization is inherently a kinetic process and the supramolecular synthon is a manifestation of these kinetic factors.

\section{Intermolecular interactions}

A very large number and variety of intermolecular interactions have been used in crystal engineering. Most notable among such interactions are hydrogen bonds that arise from the polar region of molecules and a herringbone and stacking reactions that arise from the non-polar regions of aromatic molecules and hydrophobic interaction that arise from aliphatic regions. The hydrogen bond is especially important because it is both strong and directional. ${ }^{38}$ Therefore it is a very effective design element in crystal engineering. A very large variety of hydrogen bonds are known. In addition to the stronger variety such as $\mathrm{O}-\mathrm{H} \cdots \mathrm{O}$ and $\mathrm{N}-\mathrm{H} \cdots \mathrm{O}$ there are weaker varieties like $\mathrm{C}-\mathrm{H} \cdots \mathrm{O}, \mathrm{C}-\mathrm{H} \cdots \pi$ and other varieties that incorporate metal atoms, multiple atom acceptors and other donor and acceptor groups of very feeble effectiveness. ${ }^{39}$ These hydrogen bonds have a greater or lesser effect on the outcome of the final crystal structure. Their study is a vast undertaking and the ways in which different kinds of hydrogen bonds interact with one another in crystals is in itself a topic of immense proportions. Suffice it to say that the majority of papers that one reads currently in structural crystal engineering exemplify the role of hydrogen bonds of one kind or another. Other interactions that have become popular in recent times include halogen bonds in which an electrophilic halogen atom plays the same role as the hydrogen atom does in a hydrogen bond. ${ }^{40}$ Interactions between halogen atoms such as $\mathrm{Cl} \cdots \mathrm{Cl} \mathrm{Br} \cdots \mathrm{Br}$ and I...I are special cases of halogen bonding. ${ }^{41}$ Interactions between heavy atoms such as gold, for example the aurophilic $\mathrm{Au} \cdots \mathrm{Au}$ interaction have also been used in crystal engineering. ${ }^{42}$ The general idea has been described for the ethynyl cyanobenzenes in section 2. A molecule is chosen so that there are appropriate hydrogen bond donor and acceptor groups in suitable locations of the molecule. The location and positioning of these groups is so selected such that an extended array of molecules connected with hydrogen bonds is obtained. The chicken-wire structure of the 1:1 molecular complex of cyanuric acid and melamine is a classic example of the use of hydrogen bonds in designing a layered crystal structure. $^{43}$

Interaction interference is a notable stumbling application to modular construction of a crystal structure. Accordingly interference needs to be firstly identified, then understood and finally tackled and 
even incorporated, if necessary, in the design strategy. As mentioned earlier not all crystal structures can be understood in a modular manner based on molecular functionalities. In fact the converse is almost the more common occurrence. Different types of interactions interfere with each other and the more dominant interaction (stronger and more directional) will dominate the packing and the weaker interactions are necessarily subsumed and are compromised. A number of recent papers have addressed the problem of interference. ${ }^{44}$ In optimal cases, interference may even be used in the design strategy and if a sufficiently large number of crystal structures are known, it is possible to predict more crystal structures within the structural family; this has been demonstrated in a series of geminal alkynols. ${ }^{45}$

\section{Polymorphism}

Polymorphism refers to the phenomenon wherein a given molecule exists in more than one crystal form. ${ }^{46}$ It was originally conjectured that polymorphism is the enemy of crystal engineering - it was referred to as the Nemesis of crystal engineering. ${ }^{1}$ More recent work has shown that this phenomenon is of fundamental significance in understanding crystal packing, crystal structure prediction and even in the unravelling of the mechanisms of crystallization. An additional, but by no means inconsequential reason for the importance of polymorphism is that different polymorphs of a drug molecule have been deemed in some cases to warrant separate legal protection in the form of patents. Accordingly there is a tremendous interest, in the pharmaceutical industry, to understand this phenomenon.

Polymorphism is of fundamental interest. Why should any molecule crystallize in more than one form especially when it appears that molecular recognition is such a subtle and specific event that demands an exact matching in geometrical and chemical terms of the various interacting molecules? Many reasons for polymorphism have been adduced and one of the most appealing ones is that several metastable and kinetic crystal forms may be obtained during crystallization before the most stable crystal structure, namely the thermodynamic form, appears. ${ }^{47}$ Some of these kinetic forms may be indefinitely stable. For example, although graphite is the most stable polymorph of elemental carbon, diamond which is a metastable polymorph, is indefinitely stable under ambient conditions. The outcome of crystalli- zation may lead to either or both kinetic and thermodynamic products thereby leading to polymorphism.

The study of polymorphism is a major activity in the practice of crystal engineering today. The subject may be studied through the crystallization of various crystal forms and the measurement of their properties by techniques such as single crystal X-ray crystallography, powder X-ray diffraction, differential scanning calorimetry, infrared and Raman spectroscopy and more recent more exotic techniques such as terahertz spectroscopy. From the applied viewpoint of the pharmaceutical industry it is important to be able to detect very small amounts of one polymorph in the presence of a large excess of another. Typically, the polymorph which is suspected to be present in a small amount enjoys legal protection whereas the polymorph in excess is free of patent coverage. Accordingly, an innovator company would like to show that a solid form being marketed by a generic competitor contains an infringing polymorph. Conversely, the generic company would like to show that the form which it is marketing is free of the polymorph that enjoys patent protection. All this means that there is a tremendous impetus towards making of instrumentation that can detect crystal forms at ever increasing levels of sensitivity.

Polymorphism may also be studied through computational methods. Any crystal structure is associated with energy stabilization with respect to its isolated molecules. This is the energy that must be put into a crystal in order to dissociate it into its constituent molecules, in other words the heat of sublimation. Current methods of crystal structure prediction generate a very large number of putative crystal structures associated with certain energies. ${ }^{48}$ Computed forms with the lowest energy are assumed to be ones that are obtained experimentally. These computations involve the optimization of atom-atom potentials which are empirical parameters that are related through force fields that are undergoing constant refinement and improvement. Unfortunately methods of crystal structure prediction that depend on energies of crystal structures are not quite so useful if the thermodynamic form is different from the kinetic form, and the latter is the one obtained during crystallization. ${ }^{49}$ These and other difficulties continue to engage the attention of computational chemists who are involved in the exercise of crystal structure prediction (CSP). The Cambridge Crystallographic Data Centre organizes blind 
tests in CSP every few years in which the experimental crystal structures of a few molecules are held confidentially while contestants make computational predictions about their crystal structures. ${ }^{50}$ After several months, the results of the computational groups are compared with the experimental structures. Methods of CSP have improved vastly over the past decade; one particular group obtained the correct answers for all four molecules that had been supplied for the most recent test. ${ }^{51}$ At the time of writing this review, another blind test was in progress with co-crystals, salts and polymorphs among the molecules given for the exercise.

\section{Multi-component molecular crystals}

From antiquity, crystallization has been known to be a technique of purification. Its inherent nature is that of exclusion. From a solution containing numerous compounds or a melt of several compounds, crystallization generally yields a single compound in a high state of purity. Molecular recognition is a very specific phenomenon and when a solution or melt is in a condition of supersaturation or supercooling, a kinetically controlled process that we call nucleation occurs and results in the appearance of a single component solid phase that is called a crystal. Accordingly, the idea that a crystal can contain more than one type of molecular entity is counter intuitive. Unless there are specific chemical reasons, multi-component molecular crystals are normally not expected. Within this category of substance, one may include crystals that contain two compounds both of which are normally isolated as pure crystalline solids. One may also include crystals that contain molecules of one type along with solvent molecules that are an integral part of the crystal structure, in other words they are arranged with long term periodicity and are structured regularly. It is mostly a technical nicety to distinguish between these two types of multi-component molecular crystals. $^{52}$

The first type of binary crystal has been referred to in the past as solid solution, molecular compound, addition compound, molecular complex and co-crystal. $^{53}$ The term co-crystal is usually employed to refer to a crystal in which two distinct molecular components are linked with hydrogen bonds. In these co-crystals, hydrogen bonds of the type $\mathrm{A} \cdots \mathrm{B}$ between components $\mathrm{A}$ and $\mathrm{B}$ are stronger are more specific that hydrogen bonds say of the type A $\cdots \mathrm{A}$ or $\mathrm{B} \cdots \mathrm{B}$. This is the driving force for co-crystal formation. Sometimes the acidity of the hydrogen bond donor and the basicity of the hydrogen bond acceptor are so high that proton transfer takes place across the hydrogen bond (any hydrogen bond is a case of incipient proton transfer) so that a salt is formed. ${ }^{54}$ This salt is between an organic cation and organic anion. Beyond a point, it is artificial to differentiate between a co-crystal which consists of neutral molecules and a salt which consists of ions. Other types of multi-component molecular crystals are mediated with interactions other than hydrogen bonding, say $\pi \cdots \pi$ stacking interactions and hydrophobic interactions between hydrocarbon residues. However, the term co-crystal is, rightly or wrongly, used when the two components are held together with hydrogen bonds.

Co-crystals have become particularly important because of their relevance in the pharmaceutical industry. ${ }^{55}$ In the same way that patent protection can be extended for a drug by the discovery of new polymers, such protection may also be obtained by the isolation of co-crystals between the drug and a small neutral organic molecule called a co-former. ${ }^{56}$ Co-crystallization of a drug is desirable if the co-crystals have better physical properties when compared to the parent drug. These could be better solubility, better stability or longer shelf life. Cocrystals of a drug generally have a good chance to enjoy patent protection over and above the parent drug because: (i) they are clearly new chemical entities; (ii) their design and preparation involve several elements of non-obviousness and; (iii) they generally have novel and useful properties. Generics are engaged in research aimed at generating large numbers of co-crystals for their drugs. The typical drug molecule contains a number of hydrogen bond donor and acceptor groups. Therefore these molecules are particularly amenable to the formation of (hydrogen bond based) co-crystals. The structure of the coformer for any particular drug molecule is readily obtained by the application of synthon theory. Synthons between the drug and the co-former molecules are necessarily of an unsymmetrical nature and have been termed as heterosynthons in contrast to the homosynthons, like the carboxylic acid dimer, that are obtained in the crystal structures of single component crystals. The design and properties of cocrystals is of active concern today in the practice of crystal engineering. ${ }^{57}$ 


\section{Conclusions}

Crystal engineering has completed the stages of early growth and has now entered a period of maturity and consolidated development. A considerable body of structural data has been obtained and analysed and generalizations for crystal packing are being developed with this data. New uses and applications are being suggested for crystals that are being designed systematically. For co-ordination polymers, applications such as absorption of gases and liquids is of major importance but optimization of magnetic and electric properties is also wellstudied. Design of molecular crystals for optical, electronic and chemical properties continues to be a major undertaking. Function will be of major importance in the future. The prediction of crystal structures is a major challenge and represents the cutting edge of computational crystal engineering. Unravelling the mechanism of crystallization is one of the Holy Grails of crystal engineering and several researchers are using both crystallography and noncrystallographic methods to make inroads into this complex and challenging question.

\section{Acknowledgements}

I thank the Department of Science and Technology (DST), New Delhi for the award of a J C Bose Fellowship and for supporting the India-Russia symposium on structural chemistry during which the lecture was delivered on which this article is based. I thank the Rigaku Corporation, Tokyo, for their support in the form of a tabletop single crystal diffractometer.

\section{References}

1. Desiraju G R 1989 Crystal engineering: The design of organic solids (Amsterdam: Elsevier)

2. Desiraju G R (ed.) 2003 Crystal engineering. Structure and function. Perspectives in supramolecular chemistry (Chichester: Wiley)

3. Tiekink E R and Vittal J J (eds) 2005 Frontiers in crystal engineering (Chichester: Wiley)

4. Braga D, Grepioni F and Orpen A G (eds) 1999 Crystal engineering. From molecules to crystals to materials (Dordrecht: Kluwer)

5. For some representative reviews, see: (a) Aakeröy C B 1997 Acta Crystallogr. Sect. B 53 569; (b) Goldberg I 2005 Chem. Commun. 1243; (c) Bishop R 1999 Synlett. 1351; (d) Biradha K 2003 Cryst. Eng. Comm. 5 374; (e) Aakeröy C B, Champness N R and
Janiak C 2010 Cryst. Eng. Comm. 12 22; (f) Dastidar P 2008 Chem. Soc. Ev. 37 2699; (g) Glaser R 2007 Acc. Chem. Res. 40 9; (h) Braga D and Grepioni F 2005 Chem. Commun. 3635; (i) Braga D, Brammer L and Champness N R 2005 Cryst. Eng. Comm. 7 1; Erk P, Hengelsberg H, Haddow M F and van Gelder R 2004 Cryst. Eng. Comm. 6474

6. Bragg W H 1921 Proc. Phys. Soc. (London) 3433

7. Sharma C V K 2002 Cryst. Growth Des. 2465

8. Robertson J M 1951 Proc. R. Soc. (London) Ser. A 207101

9. (a) Desiraju G R 2003 Nature 423 485; (b) Ganguly P and Desiraju G R 2010 Cryst. Eng. Comm. 12817

10. Davey R J, Dent G, Mughal R K and Parveen S 2006 Cryst. Growth Des. 61788

11. Mondal R, Howard J A K, Banerjee R and Desiraju G R 2006 Cryst. Growth Des. 62507

12. Banerjee R, Bhatt P M, Kirchner M T and Desiraju G R 2005 Angew. Chem. Int. Ed. 442515

13. Gantenberg M and Sander W 2005 Spectrochimica Acta $A 902$

14. Cohen M D and Schmidt G M J 1964 J. Chem. Soc. 1996 and the three succeeding papers

15. Pepinsky R 1955 Phys. Rev. 100971

16. Cohen M D 1987 Tetrahedron 431211

17. Herbstein F H 2005 Crystalline molecular complexes and compounds (Oxford: OUP)

18. (a) Addadi L and Lahav M $1978 \mathrm{~J}$. Am. Chem. Soc. 100 2838; (b) Leiserowitz L 1976 Acta Crystallogr. Sect. B 32127

19. (a) Paul I C and Curtin D Y 1973 Acc. Chem. Res. 6 217; (b) Curtin D Y and Paul I C 1981 Chem. Rev. 81 525; (c) Paul I C and Curtin D Y 1975 Science 18719

20. Wegner G 1969 Z. Naturforsch. 24824

21. (a) Thomas J M 1981 Nature 289 633; (b) Cohen M D, Ludmer Z, Thomas J M and Williams J O 1971 Proc. R. Soc. London Ser. A 324459

22. Ermer O $1988 \mathrm{~J}$. Am. Chem. Soc. 1103747

23. Hoskins B F and Robson R $1990 \mathrm{~J}$. Am. Chem. Soc. 1121546

24. (a) Eddaoudi M, Moler D B, Li H L, Chen B L, Reinecke T M, O'Keeffe M and Yaghi O M 2001 Acc. Chem. Res. 34 319; (b) Natarajan S and Mandal S 2004 Angew. Chem. Int. Ed. 43 4798; (c) Zhang Z P, Huang X C and Chen X M 2009 Chem. Soc. Rev. 38 2385; (d) Biradha K, Sarkar M and Rajput L 2006 Chem. Commun. 4169; (e) Baburin I A, Blatov V A, Carlucci L, Ciani G and Proserpio D M 2008 Cryst. Eng. Comm. 101822

25. Desiraju G R and Gavezzotti A 1989 J. Chem. Soc. Chem. Commun. 621

26. Desiraju G R 2007 Angew. Chem. Int. Ed. 468342

27. Dunitz J D 1991 Pure Appl. Chem. 63177

28. Lehn J-M 1988 Angew. Chem. Int. Ed. Engl. 2789

29. Desiraju G R 1995 Angew. Chem. Int. Ed. Engl. 34 2311

30. Moulton B and Zaworotko M J 2001 Chem. Rev. 101 1629

31. Whitesides G M, Simanek E E, Mathias J P, Seto C T, Chin D N, Mammen M and Gordon D M, 1995 Acc. Chem. Res. 2837 
32. Fyfe M C T and Stoddart J F 1997 Acc. Chem. Res. 30393

33. Chichak K S, Cantrill S J, Pease A R, Chiu S H, Cave G W V, Atwood J L and Stoddart J F 2004 Science 3045675

34. Mahapatra S, Azim Y and Desiraju G R 2010 J. Mol. Str. 976200

35. Dulmage W J and Lipscomb W N 1951 Acta Crystal$\log r .5260$

36. Gavezzotti A $1991 \mathrm{~J}$. Am. Chem. Soc. 1134622

37. Etter M C 1990 Acc. Chem. Res. 23120

38. Jeffrey G A 1997 An introduction to hydrogen bonding (Oxford: OUP)

39. Desiraju G R and Steiner T 1999 The weak hydrogen bond in structural chemistry and biology (Oxford: OUP)

40. Metrangolo P, Neukirch $\mathrm{H}$, Pilati $\mathrm{T}$ and Resnati $\mathrm{G}$ 2005 Acc. Chem. Res. 38386

41. (a) Pedireddi V R, Reddy D S, Goud B S, Craig D C, Rae A D and Desiraju G R $1994 J$. Chem. Soc. Perkin Trans. 2 2353; (b) Bui T T T, Dahaoui S, Lecomte C, Desiraju G R and Espinosa E 2009 Angew. Chem. Int. Ed. 48 3838; (c) Brammer L, Espallargas G M and Libri S 2008 Cryst. Eng. Comm. 101335

42. Leznoff D B, Xue B Y, Batchelor R J, Einstein F W $\mathrm{B}$ and Patrick B O 2001 Inorg. Chem. 406026

43. Simanek E E, Mammen M, Gordon D M, Chin D, Mathias J P, Seto C T and Whitesides G M 1995 Tetrahedron $\mathbf{5 1} 607$

44. (a) Tiekink E R T and Zukerman-Schpector J 2009 Cryst. Eng. Comm. 11 1176; (b) Metrangolo P, Pilati T, Terraneo G, Biella S and Resnati G 2009 ibid 11 1187; (c) Hosseinzadeh R, Lasemi Z, Seichter W and Weber E 2009 ibid 11 1331; (d) Podsiado M and Katrusiak A 2009 ibid 11 1391; (e) Santos-Contreras
R J, Martinez-Martinez J, Mancilla-Margalli A, Peraza-Campos A L, Morin-Sanchez L M, Garcia-Baez E V and Padilla-Martinez I 2009 ibid 11 1451

45. Banerjee R, Mondal R, Howard J A K and Desiraju G R 2006 Cryst. Growth Des. 6999

46. Threlfall T L 1995 Analyst 1202435

47. Desiraju G R 2002 Nature Mater. 177

48. Price S L 2009 Acc. Chem. Res. 42117

49. Sarma J A R P and Desiraju G R 2002 Cryst. Growth Des. 293

50. Day G M et al 2009 Acta Crystallogr. Sect. B 65 107

51. Neumann M A, Leusen F J J and Kendrick J 2008 Angew. Chem. Int. Ed. $\mathbf{4 7} 2427$

52. Bond A D 2007 Cryst. Eng. Comm. 9833

53. (a) Desiraju G R, 2003 Cryst. Eng. Comm. 5 466; (b) Dunitz J D 2003 Cryst. Eng. Comm. 5506

54. Aakeröy C B, Fasulo M E and Desper J 2007 Mol. Pharm. 4317

55. (a) Almarsson Ö and Zaworotko M J 2004 Chem. Commun. 1889; (b) Vishweshwar P, McMahon J A, Bis J A and Zaworotko M J 2006 J. Pharm. Sci. 95 499; (c) Childs S L and Zaworotko M J 2009 Cryst. Growth Des. 9 4208; (d) Schultheiss N and Newman A 2009 Cryst. Growth Des. 92950

56. (a) Trask A V 2007 Mol. Pharm. 4 301; (b) Stahly G P 2006 Cryst. Growth Des. 6925

57. (a) Chow K, Tong H H Y, Lum S and Chow A H L 2008 J. Pharm. Sci. 97 2855; (b) Aakeröy C B, Forbes S and Desper J $2009 \mathrm{~J}$. Am. Chem. Soc. 131 17048; (c) Martins F T, Paparidis N, Doriguetto A C and Ellena J 2009 Cryst. Growth Des. 9 5283; (d) Marivel S, Suresh E and Pedireddi V R 2008 Tetrahedron Lett. 493666 